\title{
Systemic antineutrophil cytoplasmic antibody vasculitis associated with lymphoid neoplasia
}

\author{
M A Hamidou, D El Kouri, M Audrain, J-Y Grolleau
}

\begin{abstract}
Two cases of systemic antineutrophil cytoplasmic antibody (ANCA) vasculitis in the setting of chronic lymphocytic leukaemia and angioimmunoblastic lymphadenopathy type $T$ cell lymphoma are reported. The two patients had fever of unknown origin associated with cutaneous vasculitis and "pulmonary-renal syndrome" with alveolar haemorrhage. Despite antiinfectious treatments, steroids, and chemotherapy, the vasculitis had a fatal paraneoplastic course in several weeks. When infection is excluded in patients with malignancy, atypical features should be promptly investigated for systemic vasculitis, and an ANCA test performed.

(Ann Rheum Dis 2001;60:293-295)
\end{abstract}

Rheumatic manifestations may be associated with malignancy, particularly those of the haematological type. ${ }^{1-5}$ Cutaneous vasculitis, seronegative arthritis, and polymyalgia rheumatica are the most common findings associated with myelodysplastic syndromes and lymphoid malignancies. Among lymphoid neoplasia, hairy cell leukaemia has a significant link with polyarteritis nodosa. ${ }^{4}$ Other types of systemic vasculitis are rare in the course of lymphoid malignancies ${ }^{5}$ and although antineutrophil cytoplasmic antibodies (ANCA) have not been extensively evaluated in this setting, the lymphoma associated vasculitis is generally ANCA negative. We report two original cases of fatal systemic ANCA vasculitis with pulmonary-renal syndrome during the course of aggressive $\mathrm{T}$ cell lymphoma and chronic lymphocytic leukaemia.

\section{Case reports}

PATIENT 1

Department of

Internal Medicine, University Hospital, Hôtel-Dieu, Nantes 44035, France M A Hamidou D El Kouri

J-Y Grolleau

\section{Department of} Immunology, University Hospital, Hôtel-Dieu, Nantes 44035, France M Audrain

Correspondence to: Dr Hamidou mohamed.hamidou (a) chu-nantes.fr

Accepted 28 June 2000 Creatininaemia was $120 \mu \mathrm{mol} / \mathrm{l}$ and blood nitrogen $6 \mathrm{mmol} / \mathrm{l}$. Urine analysis showed +++ red blood cells and $1.2 \mathrm{~g} /$ day glomerular proteinuria without casts. Antinuclear antibodies, rheumatoid factor, immune complexes, cryoglobulinaemia were negative, as were blood cultures, HIV, cytomegalovirus, EpsteinBarr virus, and $\mathrm{B}$ and $\mathrm{C}$ hepatitis virus serological tests. Total complement, C3, and C4 fractions were normal. A serum ANCA test was positive on indirect immunofluorescence on ethanol fixed, polymorphonuclear cells at a titre of $1 / 100$ with a cytoplasmic staining pattern (cANCA); an enzyme linked immunosorbent assay (ELISA) for anti-proteinase 3 (PR3) or anti-myeloperoxidase (MPO) antibodies was not performed. Cutaneous purpura biopsy showed leucocytoclastic vasculitis without immunoglobulin or complement deposits on direct immunofluorescence. Echocardiography was normal with no sign of endocarditis or myxoma. Lymph node examination disclosed T cell lymphoma of angio-immunoblastic lymphadenopathy type. Intravenous methylprednisolone pulses, $1 \mathrm{~g} /$ day for three days, $1 \mathrm{mg} / \mathrm{kg} /$ day prednisone, and CHOP polychemotherapy $\left(750 \mathrm{mg} / \mathrm{m}^{2}\right.$ intravenous (IV) cyclophosphamide, Adriblastine (doxorubicin), vincristine) were instituted without improvement. Bronchoalveolar lavage showed alveolar haemorrhage without bacterial, fungal, mycobacterial, viral, or Pneumocystis carinii infection, or lymphoma. He died after six weeks in respiratory and acute renal failure (creatininaemia $500 \mu \mathrm{mol} / \mathrm{l})$.

\section{PATIENT 2}

A 65 year old man presented with chronic lymphocytic leukaemia in 1981. In 1989 the leukaemia was progressing with enlarged lymph nodes. Laboratory studies showed $100 \times$ $10^{9} / 1$ lymphocytes, $30 \times 10^{9} / 1$ platelets, and haemoglobin $70 \mathrm{~g} / 1$ without haemolysis. $\gamma$ Globulin levels were $20 \mathrm{~g} / 1$ (normal range 60-140 g/1). His CHOP chemotherapy regimen included Adriblastine, vincristine, $750 \mathrm{mg} / \mathrm{m}^{2}$, IV cyclophosphamide, and prednisolone. After the second regimen of chemotherapy he was admitted to the department of haematology with a fever of unknown origin and a recent onset of dyspnoea. Clinical examination disclosed a vascular purpura of the legs with leucocytoclastic vasculitis on biopsy. Direct immunofluorescence was negative. He had maxillar sinusitis and rhinoscopy, and showed no mucosal lesions suggestive of Wegener's granulomatosis; a nasal biopsy was not performed. He had no articular or neurological symptoms. Chest radiograph and thoracic computed tomography (CT) scan showed alveolar pulmonary infiltrates, and bronchoalveolar lavage disclosed alveolar haemorrhage with $60 \%$ siderophages. There was no evidence of infection 
(pyogenic bacteria, fungal, mycobacterial and viral infections, Pneumocystis carinii) or lymphoma. Haemoglobin was $80 \mathrm{~g} / \mathrm{l}$, white blood cells were $18 \times 10^{9} / 1$ with $15 \times 10^{9} / 1 \mathrm{lym}$ phocytes, $2 \times 10^{9} / 1$ neutrophils, $0.1 \times 10^{9}$ eosinophils, platelets $30 \times 10^{9} / 1$. The serum creatinine level was $350 \mu \mathrm{mol} / 1$ and urine analysis was positive for both blood $(+++)$ and protein $(+++)$. Blood cultures, HIV test, cytomegalovirus, Epstein-Barr virus, hepatitis B and C, antinuclear antibodies, rheumatoid factor, immune complexes, and cryoglobulinaemia were negative. Total complement, C3, and C4 fractions were normal. Renal biopsy was not performed because of the low platelet count. Bone marrow analysis, brain, thoracic, abdominal CT scan, and cerebrospinal fluid analysis disclosed either histologically aggressive transformation of chronic lymphocytic leukaemia, or infection. Digestive fibroscopy did not show solid neoplasia. By indirect immunofluorescence on serum, ANCA was positive at a titre of $1 / 1000$ with a cytoplasmic staining pattern; an ELISA for anti-PR3 and anti-MPO antibodies was not performed. Wide spectrum antibiotic treatment, IV amphotericin B, IV acyclovir, and antituberculous treatment were given without success for this refractory unexplained fever. After eight weeks his condition deteriorated and despite treatment with intravenous methylprednisolone, the patient died.

\section{Discussion}

The association between lymphoma and vasculitis is rare, ${ }^{1-3}$ and the most common clinical vasculitic manifestation is cutaneous vasculitis without systemic involvement. The relation between the two conditions is unclear. The vasculitis may appear after the malignancy, at the time of diagnosis of the malignancy, or it may precede the neoplasm. The paraneoplastic characteristic of vasculitis is not constant, and generally the vasculitis evolves without influence of malignancy or treatment. The mechanisms of the vasculitis associated with malignancy are complex, including formation of immune complexes, polyclonal activation of $\mathrm{B}$ lymphocytes, monoclonal immunoglobulin activity, antibodies directed toward endothelial antigens, direct effect of the malignancy on the vascular wall or adverse reactions to anticancer treatment. ${ }^{12}$ CD5 positive B cells present in certain lymphoproliferative disorders as chronic lymphocytic leukaemia, may have a role in these mechanisms, ${ }^{6}$ producing autoantibodies and monoclonal immunoglobulins with various autoantibody activities.

In these two case reports the association between vasculitis and lymphoid neoplasia did not seem coincidental. The two patients had clinical conditions suggestive of disseminated vasculitis, affecting small sized vessels, ${ }^{7}$ with proven leucocytoclastic cutaneous vasculitis, alveolar haemorrhage, and nephritis (so called "pulmonary-renal syndrome"). We found no opportunistic infection or malignant tissue infiltration. Despite treatment with steroids and alkylating agents in one patient, and steroids alone in the other, the vasculitis had an unusual fulminant course, ${ }^{8}$ at the same time as lymphoid neoplasia, suggesting a paraneoplastic mechanism. The two patients had high titre ANCA by indirect immunofluorescence test, with cytoplasmic staining pattern, and without cryoglobulinaemia, monoclonal gammopathy, other autoantibodies (rheumatoid factor, antinuclear antibodies, etc), or circulating immune complexes, suggesting natural polyreactive antibodies. Also, the ANCA positivity did not seem artefactual. Indeed, in a prospective study from 1995 to 1998 we determined the prevalence of ANCA positivity in 140 chronic lymphoid malignancies, ${ }^{9}$ and found only two positive serum samples with low titre perinuclear ANCA (chronic lymphocytic leukaemia and Waldenström's disease) and one with atypical ANCA (lymphoma). None of these patients had clinical manifestations of vasculitis. The global prevalence of the ANCA test in this study was $2 \%$, similar to the $0-1.8 \%$ ANCA positivity in the French hospital population. ${ }^{10}$

Among lymphoid malignancies, the association between hairy cell leukaemia and leucocytoclastic vasculitis, polyarteritis nodosa, or temporal arteritis is the most common. ${ }^{4}$ In 1991 Fain et al found 23 reported observations of vasculitis associated with lymphoma: 15 patients had non-Hodgkin's lymphoma and eight had Hodgkin's disease. ${ }^{3}$ Most patients had isolated cutaneous vasculitis, and systemic vasculitis was generally of the polyarteritis nodosa type, affecting predominantly medium and small sized arteries.

To our knowledge the association between lymphoid malignancies and paraneoplastic systemic ANCA vasculitis has never been reported. Tatsis et al, in a retrospective analysis of 477 patients with Wegener's granulomatosis, found a significant relation with malignancy, in particular with renal carcinoma, but not with lymphoma. ${ }^{11}$ During the course of Wegener's granulomatosis, mediastinal mass and hilar adenopathy, simulating lymphoma, ${ }^{12}$ are uncommon findings. One case report of microscopic polyangiitis associated with nonHodgkin's lymphoma was described, but the ANCA results were not reported. ${ }^{13}$ Gratadour et al reported the occurrence of classic Wegener's granulomatosis nine months after remission of Hodgkin's disease, ${ }^{14}$ and Davenport reported the association of a positive cANCA leucocytoclastic vasculitis, and clinical findings, leading to a misdiagnosis of Wegener's granulomatosis. ${ }^{15}$ A postmortem examination disclosed a non-Hodgkin's lymphoma without granulomatous vasculitis. Zuckerman et al described positive cANCA in a patient with lymphoma without vasculitis, ${ }^{16}$ and Savige et al found cANCA in two of 17 patients with lymphoproliferative syndromes. ${ }^{17}$ Esnault et al found ANCA in 12 serum samples of 125 $(10 \%)$ patients with monoclonal component and concluded that there was non-specific autoreactivity. ${ }^{18}$

In conclusion, we report an unusual association between systemic ANCA vasculitis and lymphoid malignancies. Despite steroids and chemotherapy, the vasculitis had a particularly aggressive paraneoplastic course. In patients with malignancy, when infection or disease 
relapse are ruled out, atypical features should be promptly investigated for systemic vasculitis. ANCA may provide an useful tool for diagnosis.

1 Naschitz JE, Rosner I, Rozenbaum M, Elias N, Yeshurun D. Cancer-associated rheumatic disorders. Semin Arthritis Rheum 1995;24:231-41.

2 Mertz LE, Conn DL. Vasculitis associated with malignancy. Curr Opin Rheumatol 1992;4:39-46.

3 Fain O, Guillevin L, Kaplan G, Sicard D, Lemaire V, Godeau P, et al. Vascularites et néoplasies. Quatorze observations. Ann Med Interne 1991;142:486-504.

4 Hasler P, Kistler H, Gerber H. Vasculitides in hairy cell leukemia. Semin Arthritis Rheum 1995;25:134-42.

5 Wooten MD, Jasin HE. Vasculitis and lymphoproliferative diseases. Semin Arthritis Rheum 1996;26:564-74

6 Youinou P, Mackenzie LE, Lamour A, Mageed RA, Lydyard PM. Human CD5-positive B cells in lymphoid malignancy and connective tissue diseases. Eur J Clin Invest 1993;23: and conn 1390.

7 Jennette JC, Falk RJ. Small-vessel vasculitis. N Engl J Med 1997;337:1512-23.

8 Guillevin L, Cordier J-F, Lhote F, Cohen P, Jarrousse B, Royer I, et al. A prospective multicenter randomized trial comparing steroids and pulse cyclophosphamide versus steroids and oral cyclophosphamide in the treatment of generalized Wegener's granulomatosis. Arthritis Rheum 1997;40:2187-98.

9 Hamidou MA, Derenne S, Audrain M, Berthelot JM, Boumalassa A, Grolleau JY. Prevalence of rheumatic manifestations and ANCA in haematologic neoplasia. A prospective study. Rheumatology 2000;39:417-20.
10 Maillefert JE, Pfitzenmeyer P, Thenet M, Olsson NO, Piroth C, Behin A, et al. Prevalence of ANCA in a hospitalized elderly French population. Clin Exp Rheumatol 1997;15: 603-7.

11 Tatsis E, Reinhold-Keller E, Steindorf K, Feller AC, Gross WL. Wegener's granulomatosis associated with renal cell carcinoma. Arthritis Rheum 1999;42:751-6.

12 George TM, Cash JM, Farver C, Sneller M, van Dyke CW, Derus CL, et al. Mediastinal mass and hilar adenopathy. Rare thoracic manifestations of Wegener's granulomatosis. Arthritis Rheum 1997;40:1992-7.

13 Garcia Parès D, Vallès M, Bronsoms J, Torguet P, Maté G, Mauri JM, et al. Microscopic polyangiitis associated with non-Hodgkin's lymphoma. Nephrol Dial Transplant 1999; $14: 1038-9$.

14 Gratadour P, Fouque D, Laville M, Fourcade J, Ffrench M, Colon S, et al. Wegener's granulomatosis with antiproteinase-3 antibodies occurring after Hodgkin's disease. Nephron 1993;64:456-61.

15 Davenport A. " False positive " perinuclear and cytoplasmic anti-neutrophil cytoplasmic antibody results leading to misdiagnosis of Wegener's granulomatosis and/or microscopic polyarteritis. Clin Nephrol 1992;37:124-30.

16 Zuckerman KK, Leventhal L, Wynne C. Positive c-ANCA in a patient with lymphoma and without vasculitis. J Clin Rheumatol 1997;3:279-81.

17 Savige JA, Chang L, Smith CL, Duggan JC. Anti-neutrophil cytoplasmic antibodies (ANCA) in myelodysplasia and other haematologic disorders. Aust NZ J Med 1994;24: other 7 .

18 Esnault VLM, Jayne DRW, Keogan MT, Brownlee AA, Testa A, Lecarrer D, et al. Anti-neutrophil cytoplasmic antibodies in patients with monoclonal gammopathies. J Clin Lab Immunol 1990;32:153-9. 